\title{
A simple robust control for simulated moving bed chromatographic separation
}

\author{
Paul Suvarov*, Alain Vande Wouwer*, Achim Kienle** \\ * Service d'Automatique, Université de Mons (UMONS), \\ B-7000 Mons, Belgium (e-mail: Paul.Suvarov@umons.ac.be; Alain.VandeWouwer@umons.ac.be) \\ ** Max-Planck-Institut für Dynamik komplexer technischer Systeme, \\ D-39106 Magdeburg, Germany (e-mail:Kienle@mpi-magdeburg.mpg.de)
}

\begin{abstract}
Simulated moving bed (SMB) is a continuous chromatographic process used for the separation of chemical mixtures. This paper presents an almost plug-and-play control strategy, which requires little prior knowledge about the adsorption properties. A discrete-time nonlinear model derived from the wave theory is the basis to control the position of the adsorption and desorption fronts. The front velocities are estimated on-line enabling the compensation of initial parameter discrepancies, and drift due to adsorbent aging or temperature fluctuation. The overall performance of the control strategy is evaluated using numerical simulation for two case studies relative to the separation of fructo-oligosaccharides and cyclopentanone - cycloheptanone, respectively.
\end{abstract}

Keywords: simulated moving bed, chromatography, control, adaptive, parameter estimation

\section{INTRODUCTION}

Simulated Moving Bed (SMB) chromatographic separation processes were first used in large scale production by universal oil products (UOP) in the early 60's (Broughton and Gerhold, 1961). SMB processes (Fig. 1.) allow the continuous separation of binary mixtures and are discretized versions of the ideal True Moving Bed (TMB) processes. Following the successful operation in hydrocarbon and sugar separation, the technology was improved and increasingly applied to the separation of fine chemicals, pharmaceuticals, forensic samples and others.

A schematic view of a typical process is presented in figure 1.The inputs (feed mixture and solvent) and outputs (extract and raffinate) divide the system in four zones each containing one or more chromatographic columns, depending on the separation being performed. Pumps connected at each port determine the liquid phase flow rates in all the zones.

The feed mixture composed of components $\mathrm{A}$ and $\mathrm{B}$ is injected between zone II and III. The adsorbent is chosen in such a way that the two components are adsorbed at different rates, allowing them to travel with different velocities. The less adsorbed component (A) is collected at the raffinate port and the more adsorbed one (B) at the extract port. The separation of the two components is performed in zone II and III, whereas zones I and IV are dedicated to adsorbent regeneration and solvent recycling, respectively, for total separation cases.

The liquid-solid counter-current movement is obtained by rotating the columns containing the adsorbent in the opposite direction to the liquid phase flow. This task can be achieved by physically rotating the columns in the opposite direction to the liquid flow or by switching the valves connecting the pumps to the system, by one column length in the direction of the liquid flow. A precise equilibrium between the velocities of the liquid and solid phases must be maintained in each zone, to ensure that the fronts of the internal concentration profiles remain in their designated zones.

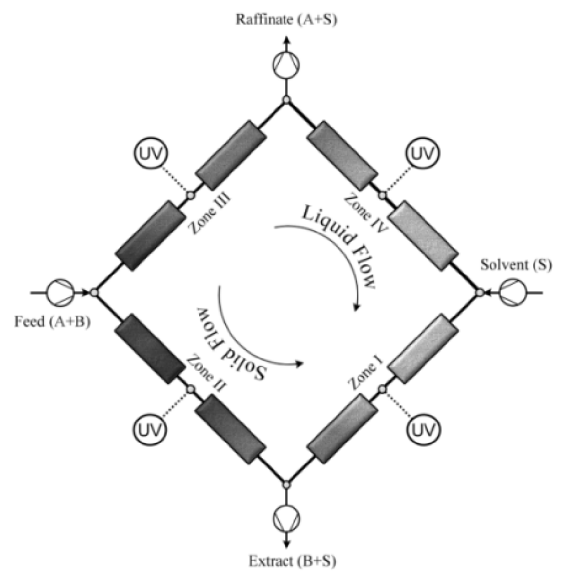

Fig. 1. SMB configuration

These operating points can be obtained using the triangle theory (Storti et al., 1993) (Mazzotti et al., 1997). The various degrees of freedom of the SMB process can be exploited to improve productivity, such as the variation of the column configuration (VariCol) (Ludemann-Hombourger et al., 2000), (Toumi et al., 2003), (Zhang et al., 2003), modulation of the flow-rates (PowerFeed), (Zhang et al., 2003; 2004), pulse modulated feed input (I-SMB) (Teshima et al., 1995), feed concentration modulation (ModiCon) (Schramm et al., 2003), or the optimization of all these parameters (SMB superstructure) (Kawajiri and Biegler, 2006; 2008).

Besides the selection of optimal operating conditions, the regulation of the process in order to reject potential disturbances has also attracted considerable attention, e.g. inferential PI controller (Schramm, Gröner and Kienle, 2003), the 'cycle to cycle' control concept based on model predictive control (Grossmann et al., 2008), (Erdem et al., 
2005), (Song et al., 2006), (Engell and Toumi, 2005).

In (Fütterer, 2008), a simple adaptive control scheme has been proposed in the case of linear adsorption isotherms, which is based on a discrete-time model of the front wave position and an adaptation scheme for the wave velocity. This control scheme has the advantage of simplicity, as even a proportional controller can be sufficient in most cases, but also to require little prior knowledge about the adsorption properties (isotherm parameters), and to be self-optimizing as it allows the concentration fronts to be moved in optimal locations. In the present study, the control concept is further analysed for the case of linear and nonlinear adsorption isotherms based on two case studies, i.e. the separation of Fructo-OligoSaccharides (FOS) and cyclopentanone cycloheptanone. Extensive simulation tests are conducted to investigate the control performance and robustness. Further research directions are discussed including the design of an observer of the front wave position so as to reduce the requirement on the availability of UV detectors.

The paper is organized as follows. The next section describes the mass-balance partial differential equation (PDE) model of the SMB plant. In the third section, a discrete-time model of the location of the foot point of the concentration fronts is derived. This model is used in section 4 to design a simple adaptive control scheme, which is analysed in depth in section 5. Conclusions and prospects are given in section 6 .

\section{SMB MODELLING AND SIMULATION}

For the typical SMB unit of figure 1, with 2:2:2:2 column configuration, a system of 32 mass balance PDEs (1-2) are used. The mass transfer between the two phases is modelled using a Linear Driving Force model (2).

$$
\begin{aligned}
& \frac{\partial C_{i, j}}{\partial t}+F \frac{\partial q_{i, j}}{\partial t}+v_{j} \frac{\partial C_{i, j}}{\partial z}=D_{j} \frac{\partial^{2} C_{i, j}}{\partial z^{2}}, i=A, B ; j=1 \ldots 8 \\
& \frac{\partial q_{i, j}}{\partial t}=K_{i}\left(q_{i, j}^{e q}-q_{i, j}\right)
\end{aligned}
$$

where $C_{i, j}$ and $q_{i, j}$ represent the concentrations of the two components in the liquid and solid phase, $F$ is the phase ratio, $v_{j}$ is the speed of the liquid phase, $D_{j}$ is the axial dispersion coefficient, $K_{i}$ is the mass transfer coefficient, $i$ identifies the component in the feed mixture and $j$ the column.

The equilibrium between the two phases is described by a linear isotherm (3) for the FOS separation and by a competitive Langmuir isotherm (4) for the cyclopentanone cycloheptanone separation.

$q_{i, j}^{e q}=H_{i} C_{i, j}, i=A, B ; j=1, \ldots, 8$

$q_{i, j}^{e q}=\frac{H_{i} C_{i, j}}{1+b_{A} C_{A, j}+b_{B} C_{B, j}}, i=A, B ; j=1, \ldots, 8$

where $H_{i}$ and $b_{i}$ are the Henry coefficients and the adsorption equilibrium constants respectively.

Dirichlet boundary conditions are considered at the input of each column. For the columns which are not at the feed or solvent ports, these conditions simply express concentration continuity (5).

$$
C_{i, j+1}(0, t)=C_{i, j}(L, t), i=A, B ; j=1,2,3,5,6,7
$$

whereas for the columns at the solvent or feed ports, the conditions are:

$$
\begin{aligned}
& C_{i, 1}(0, t)=C_{i, 8}(L, t) \frac{v_{I V}}{v_{I}}, i=A, B \\
& C_{i, 5}(0, t)=\frac{v_{I I} C_{i, 4}(L, t)+v_{F e} C_{i, F e}}{v_{I I I}}, i=A, B
\end{aligned}
$$

where $L$ is the length of the column, $v_{I \ldots I V}$ is the liquid phase velocity in each zone, $v_{F e}$ and $C_{i, F e}$ are the velocity and concentrations of the two components in the feed mixture injected.

To alleviate possible spurious numerical oscillations in presence of steep moving concentration fronts, zerodispersion and zero-adsorption conditions are expressed at the output of each column, using a simple advection equation. (Haag et al., 2001 and Schiesser, 1996)

$$
\frac{\partial C_{i, j}(L, t)}{\partial t}=-v_{j} \frac{\partial C_{i, j}(L, t)}{\partial z}
$$

The counter-current movement needed for the separation is obtained by rotating the columns in the opposite direction of the liquid phase while the boundary conditions are fixed.

The SMB model is simulated using a method of lines approach with finite elements and quadratic basis functions for spatial discretization, and a stiff differential ordinary differential equation solver (as available in Matlab and the MatMol library (www.matmol.org; Wouwer et al., 2004).

\section{WAVE FRONT MODELLING}

In order to control the purities at the outputs, the position of the waves forming the inner concentration profiles must be known. The position of the waves in each zone can be controlled by adjusting the external flow rates and the time duration of the commutation cycle $T_{S W}$. In this section, a model describing the movement of the wave within one zone is derived and used to set-up a one-cycle ahead predictor. UV detectors are assumed available in the middle of each zone as shown in figure 1. The foot point of a concentration wave is determined at the intersection of the wave profile with a threshold value as shown in figure 2 . This threshold has to be adjusted with respect to the UV detector sensitivity and the presence of noise.

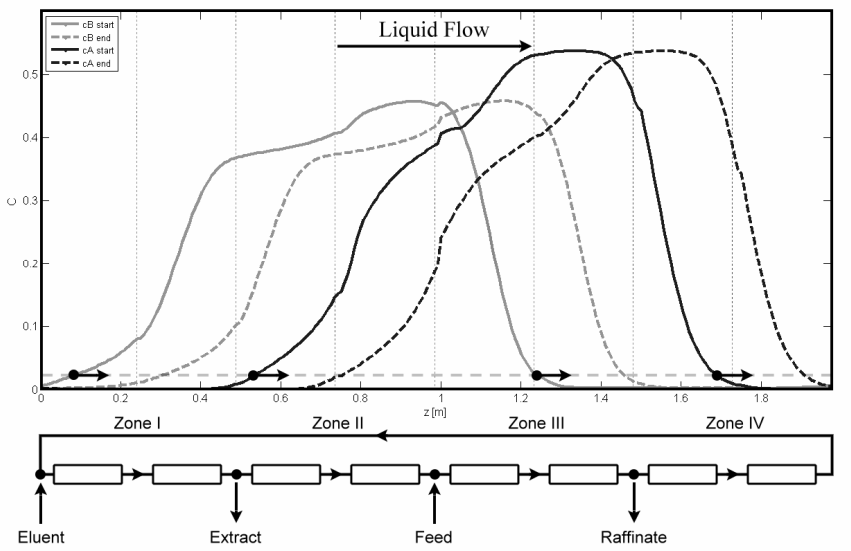

Fig. 2. Concentration profiles inside the SMB at cyclic steady state, for the cyclopentanone - cycloheptanone separation. 
It is further assumed that the velocity of the wave $v_{w}$ is constant during one cycle. It is directly proportional to the flow rate in the corresponding zone.

The travelling time of the wave from its initial position to the UV detector - located halfway in the zone - can be determined from the measurements, and in turn the position of the wave (fig. 3 and equations 9-10). This value is normalized with respect to the duration of the cycle, and is referred to as the normalized retention time $(\tau)$.

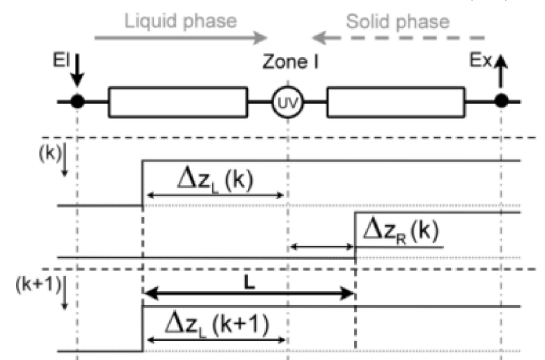

Fig. 3. Foot-point movement during one cycle.

$$
\begin{aligned}
\Delta z_{L}(k) & =v_{w} T_{S W} \tau \\
\Delta z_{R}(k) & =v_{w} T_{S W}(1-\tau)
\end{aligned}
$$

When commutation occurs the wave front is shifted by one column length.

$\Delta z_{L}(k+1)=L-\Delta z_{R}(k)$

The wave velocity is assumed proportional to the fluid flow rate and inversely proportional to the $\theta$ parameter which is dependent on the adsorbent properties.

$v_{w}=Q \frac{L}{\theta}$

For linear isotherms, the parameters can be obtained from the characteristic speed of the waves.

$$
\begin{aligned}
& \theta_{I}=\theta_{I I I}=\frac{A L\left(H_{B} F+1\right)}{F+1} \\
& \theta_{I I}=\theta_{I V}=\frac{A L\left(H_{A} F+1\right)}{F+1}
\end{aligned}
$$

For nonlinear isotherms (4), equations (13-14) can be extended by considering the flow rates corresponding to the optimal operating point determined using the triangle theory (generalized to the isotherms under consideration).

$\theta_{i}=\frac{A L\left(m_{j} F+1\right)}{F+1}, i=I, \ldots, I V \quad \theta_{i}=Q_{i} T_{S W}$

A one-cycle ahead predictor of the retention time is given by:

$\hat{\tau}_{i}(k+1)=\frac{\theta_{i}-Q_{i}(k)\left(1-\tau_{i}(k)\right) T_{S W}(k)}{Q_{i}(k+1) T_{S W}(k+1)}, i=I, \ldots, I V$

\section{CONTROL DESIGN AND PARAMETER ESTIMATION}

The parameters $\theta_{i}$ can be seen as the optimal open loop inputs to the process.

$\theta_{i}=Q_{i} T_{S W}$

To face perturbations, it is necessary to introduce a feedback control. For illustration purposes the following simple proportional control law is considered:

$w_{i}(k)=\theta_{i}\left(1-K\left(y_{R E F, i}-y_{i}(k)\right)\right), i=I, \ldots, I V$ where $y_{i}(k)$ are the measured residence times and $y_{R E F, i}$ the set-points.

As the feed flow rate is imposed by the production requirements, the cycle duration is adapted accordingly.

$T_{S W}(k)=\frac{w_{I I I}(k)-w_{I I}(k)}{Q_{F e}^{*}}$

The internal/external flow rates are then computed

$Q_{i}=\frac{w_{i}(k)}{T_{S W}(k)}$

$Q_{E l}=Q_{I}-Q_{I V} ; Q_{E x}=Q_{I}-Q_{I I} ; Q_{R a}=Q_{I I I}-Q_{I V}$

The manipulated variables $u_{i}(k)$ are therefore the four external flow-rates and the cycle duration.

It is also possible to incorporate constraints on the maximum flow rates. If one of the following inequality constraints is violated, the cycle duration is determined from the corresponding maximum flow rate. In this case it is necessary to accept a variation of the feed flow rate.

$$
\begin{array}{ll}
\frac{w_{I}(k)}{Q_{I, \max }} \leq T_{S W}(k), & \frac{w_{I}(k)-w_{I I}(k)}{Q_{E x, \max }} \leq T_{S W}(k) \\
\frac{w_{I I I}(k)-w_{I I}(k)}{Q_{F e, \max }} \leq T_{S W}(k), & \frac{w_{I I I}(k)-w_{I V}(k)}{Q_{R a, \max }} \leq T_{S W}(k)
\end{array}
$$

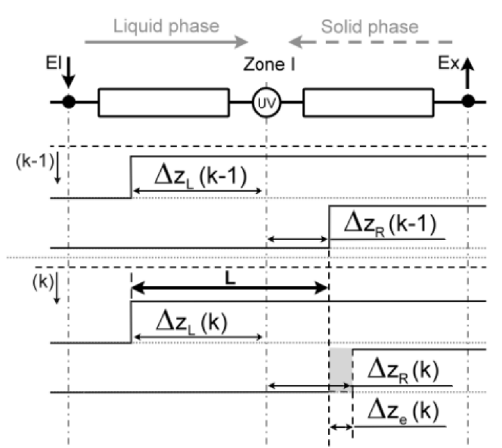

Fig. 4. Effect of a perturbation on the wave movement.

Variations of the column parameters have an important impact on the position of the waves like illustrated in figure 4. The $\theta_{i}$ parameters, containing lumped in all the adsorption parameters, are adjusted online by means of a parameter estimation algorithm. The wave front displacement $\Delta z_{e}(k)$ is translated into retention time error $\tau_{e}(k)$ using the wave front model, and then used to compute parameter errors (24) and a new set of parameters for the next cycle (25).

$\tilde{\theta}_{i}(k)=Q_{i}(k) T_{S W}(k) \tau_{e}(k)$

$\hat{\theta}_{i}(k+1)=\hat{\theta}_{i}(k)+\left(1-K_{\theta}\right) \tilde{\theta}_{i}(k), 0 \leq K_{\theta} \leq 1$

The control strategy is summarized in figure 5 .

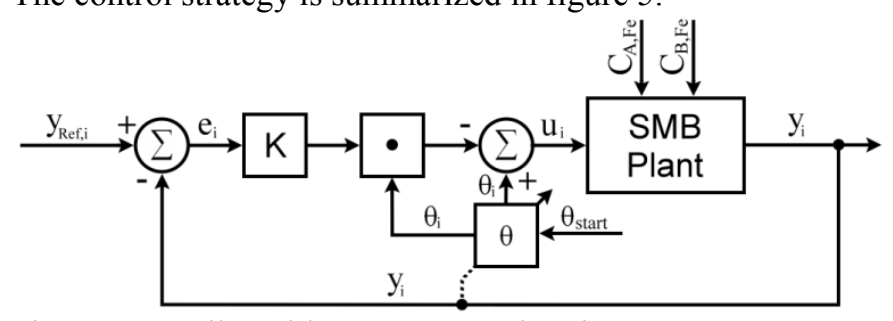

Fig. 5. Controller with parameter estimation 


\section{CONTROL PERFORMANCE AND ROBUSTNESS}

Extensive tests are now carried out to assess the performance and robustness of the control strategy in two case studies, i.e., fructo-oligosaccharides and cyclopentanone - cycloheptanone separations, respectively. The parameters of these two processes are given in Tables 1-2 (Nobre et al., 2012) (Grosfils et al., 2007). Parameter changes can occur due to adsorbent aging or temperature fluctuation and affect the velocity of the travelling waves, resulting in a runaway from the desired set-points. Changes in the composition of the feed mixture have a similar effect on the position of the waves.

Several performance indicators are now defined. Mean values of the concentration profiles (26) at both outlets are used to detect when the plant reaches cyclic steady state, and evaluate the start-up time. Purities (27), productivity (28) and solvent consumption (29) are evaluated for the remaining part of the simulation.

$$
\begin{aligned}
& m C_{i, k}=\frac{1}{T_{S W}} \int_{t}^{t+T_{S W}} C_{i, k}(t) d t, i=A, B ; k=E x, R a \\
& P_{i, k}=\frac{1}{t_{\text {end }}-t_{\text {start }}} \int_{t_{\text {start }}}^{t_{\text {end }}} \frac{C_{i, k}(t)}{C_{A, k}(t)+C_{B, k}(t)} d t \\
& P R_{i, k}=\frac{1}{t_{\text {end }}-t_{\text {start }}} \frac{C_{i, F e}}{(1-\varepsilon) V_{T}} \int_{t_{\text {sart }}}^{t_{\text {end }}} Q_{k}(t) d t \\
& S C=\frac{1}{t_{\text {end }}-t_{\text {start }}} \frac{C_{E l}}{C_{F e}} \int_{t_{\text {start }}}^{t_{\text {end }}} \frac{Q_{E l}(t)+Q_{F e}(t)}{Q_{F e}(t)} d t
\end{aligned}
$$

where $C_{i, k}$ is the concentration of the two components at the outlets, $C_{i, \mathrm{Fe}}$ the concentration of the two components at the feed input, $C_{F e}$ the total feed concentration, $C_{E l}$ the eluent concentration, $T_{S W}$ the duration of the cycle, $t_{\text {start }}$ the start-up time-stamp, $t_{\text {end }}$ the end of simulation timestamp, $V_{T}$ the total volume of the columns in the system, $Q_{[E l ; E x ; F e ; R a]}$ are the external flow-rates.

Table 1. Column parameters - fructo-oligosaccharide separation;

\begin{tabular}{|l|l|l|}
\hline \multirow{4}{*}{$\begin{array}{c}\text { Column } \\
\text { parameters }\end{array}$} & length & $0.248 \mathrm{~m}$ \\
\cline { 2 - 3 } & diameter & $0.016 \mathrm{~m}$ \\
\cline { 2 - 3 } & porosity & 0.3784 \\
\cline { 2 - 3 } & discretization points & 16 \\
\cline { 2 - 3 } & $\mathrm{HA}$ & 0.3954 \\
\cline { 2 - 3 } & $\mathrm{HB}$ & 0.0251 \\
\hline$C_{A, F e}$ & $85 \mathrm{mg} / \mathrm{ml}$ \\
\hline$C_{B, F e}$ & $64 \mathrm{mg} / \mathrm{ml}$ \\
\hline Desired feed flow-rate & $6 \mathrm{ml} / \mathrm{min}$ \\
\hline Foot-point threshold & $2.5 \mathrm{mg} / \mathrm{ml}$ \\
\hline Set-points & {$[0.80 .80 .20 .2]$} \\
\hline Controller gain $(K)$ & 0.20 \\
\hline Estimator gain $\left(K_{\theta}\right)$ & 0.65 \\
\hline
\end{tabular}

Table 2. Column parameters used in the case of cyclopentanone - cycloheptanone separation;

\begin{tabular}{|l|l|l|}
\hline \multirow{4}{*}{$\begin{array}{c}\text { Column } \\
\text { parameters }\end{array}$} & length & $0.25 \mathrm{~m}$ \\
\cline { 2 - 3 } & diameter & $0.02 \mathrm{~m}$ \\
\cline { 2 - 3 } & porosity & 0.83 \\
\cline { 2 - 3 } & discretization points & 16 \\
\cline { 2 - 3 } & $\mathrm{HA}$ & 5.97 \\
\cline { 2 - 3 } & $\mathrm{HB}$ & 8.52 \\
\cline { 2 - 3 } & $\mathrm{bA}$ & $0.154 \mathrm{vol} \%^{-1}$ \\
\cline { 2 - 3 } & $\mathrm{bB}$ & $0.295 \mathrm{vol} \%^{-1}$ \\
\hline$C_{A, F e}$ & $0.5 \mathrm{vol} \%$ \\
\hline$C_{B, F e}$ & $0.5 \mathrm{vol} \%$ \\
\hline Desired feed flow-rate & $7.59 \mathrm{ml} / \mathrm{min}^{-1}$ \\
\hline Foot-point threshold & $0.05 \mathrm{vol} \%$ \\
\hline Set-points & $0.30 .60 .60 .7]$ \\
\hline Controller gain $(K)$ & 0.10 \\
\hline Estimator gain $\left(K_{\theta}\right)$ & 0.90 \\
\hline
\end{tabular}

\subsection{Plant model mismatch}

In this experiment, start-up from wrong operating conditions as illustrated in figures 6-7 is considered. This scenario mimics an initial plant design which is based on erroneous Henry constants (up to 50\%) and which is automatically corrected by the control algorithm. For each operating point in the mesh an open-loop and an closed-loop simulation is performed, starting with fully regenerated columns.

The final operating points are concentrated in the close vicinity of the optimal operating point.
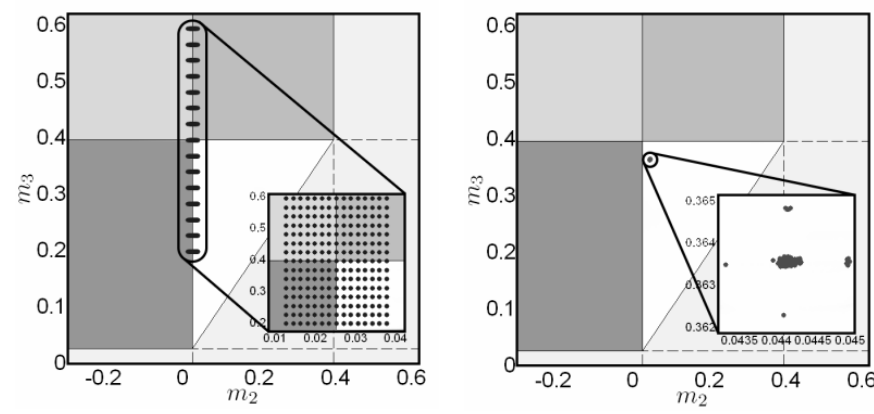

Fig. 6. Initial (left) and final (right) $\mathrm{m} 2 / \mathrm{m} 3$ operating points FOS separation
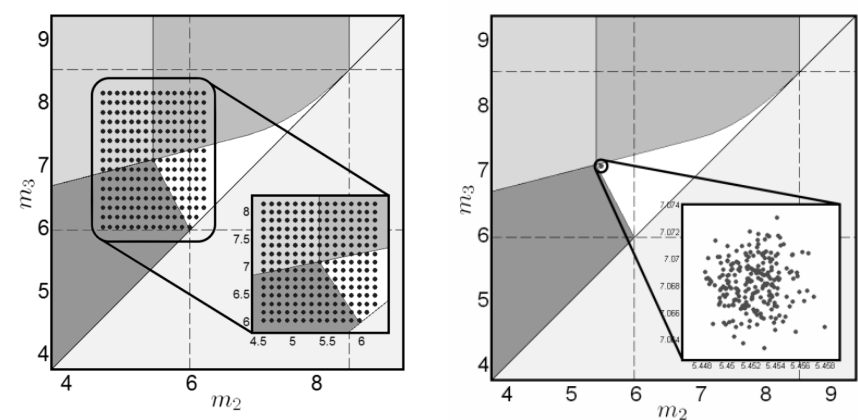

Fig. 7. Initial (left) and final (right) $\mathrm{m} 2 / \mathrm{m} 3$ operating points cyclopentanone - cycloheptanone separation. 
The performance indicators, obtained at the end of each simulation, are presented in figures 8-9 and tables 3-4. It can be observed that the controller speed-ups the plant start-up, and maximizes the total productivity and purities for the two separations, demonstrating the good robustness of the scheme independently of the linearity of the isotherms.
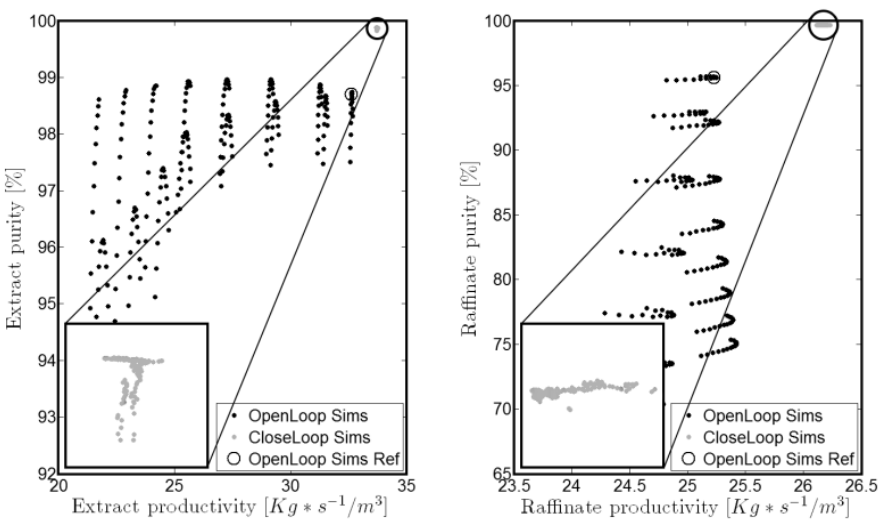

Fig. 8. Productivity versus purity - fructo-oligosaccharide separation

Table 3. Start-up time and solvent consumption for FOS separation;

\begin{tabular}{|c|c|c|c|c|c|}
\hline \multicolumn{2}{|c|}{$\begin{array}{c}\text { Performance } \\
\text { Parameter }\end{array}$} & Min. & Mean & Max. & Variance \\
\hline $\begin{array}{c}\text { Start-up } \\
\text { time [min] }\end{array}$ & OL & 29.08 & 48.12 & 75.68 & 174 \\
\cline { 2 - 6 } & CL & 26.19 & 29.59 & 35.37 & 1.68 \\
\hline $\begin{array}{c}\text { Solvent } \\
\text { consumption }\end{array}$ & OL & 13.42 & 13.42 & 13.42 & $3.07 \mathrm{e}^{-27}$ \\
\cline { 2 - 6 } & CL & 15.49 & 15.50 & 15.54 & $1.31 \mathrm{e}^{-4}$ \\
\hline
\end{tabular}
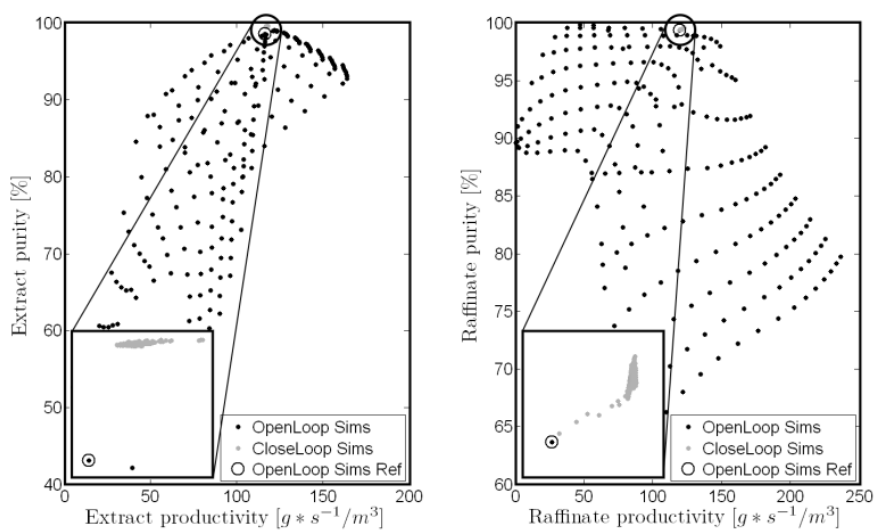

Fig. 9. Productivity versus purity - cyclopentanone cycloheptanone separation.

Table 4. Start-up time and solvent consumption for cyclopentanone - cycloheptanone separation;

\begin{tabular}{|c|c|c|c|c|c|}
\hline \multicolumn{2}{|c|}{$\begin{array}{c}\text { Performance } \\
\text { Parameter }\end{array}$} & Min. & Mean & Max. & Variance \\
\hline $\begin{array}{c}\text { Start-up } \\
\text { time }[\mathrm{min}]\end{array}$ & OL & 105.1 & 391.9 & 795.7 & $2.432 \mathrm{e}^{4}$ \\
\cline { 2 - 6 } & CL & 181.8 & 250.2 & 275.2 & 282 \\
\hline $\begin{array}{c}\text { Solvent } \\
\text { consumption }\end{array}$ & OL & 16.31 & 21.02 & 119.8 & 97.7 \\
\cline { 2 - 6 } & CL & 20.09 & 20.12 & 20.14 & $5.89 \mathrm{e}^{-5}$ \\
\hline
\end{tabular}

\subsection{Disturbance rejection}

When a disturbance affects the feed mixture composition, shock or expansion waves appear which change the concentration wave amplitude and position. Henry coefficients can also be affected due to adsorbent aging or temperature fluctuation. These changes affect the wave velocities and as a result their position.

As a test example, a severe decrease in Henry coefficients of $20 \%$ is injected at cycle 70 , well after the plant has reached cyclic steady state. In figures $10-11$, the region where total separation is achieved after the change in Henry coefficients is represented with dotted lines. The plant is started at the operating point 1 , reaches cyclic steady state before and after the disturbance is injected at points 2 and 3 respectively. The operating point of the plant is dragged inside the area of the triangle formed by the new Henry coefficients close to the optimal point.
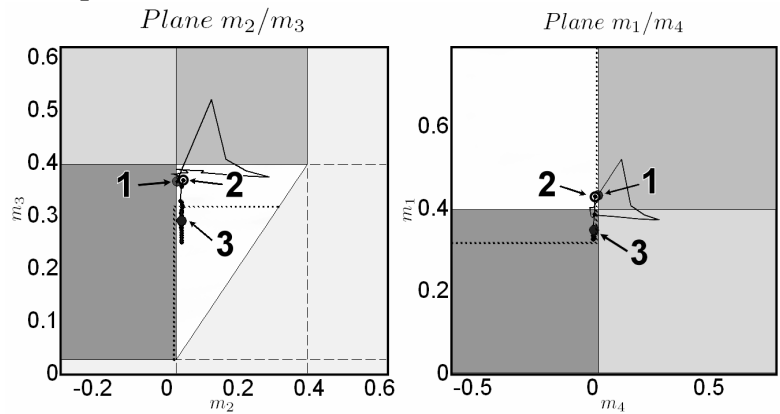

Fig. 10. Response of the system to a disturbance in Henry coefficients - Fructo - oligosaccharide separation
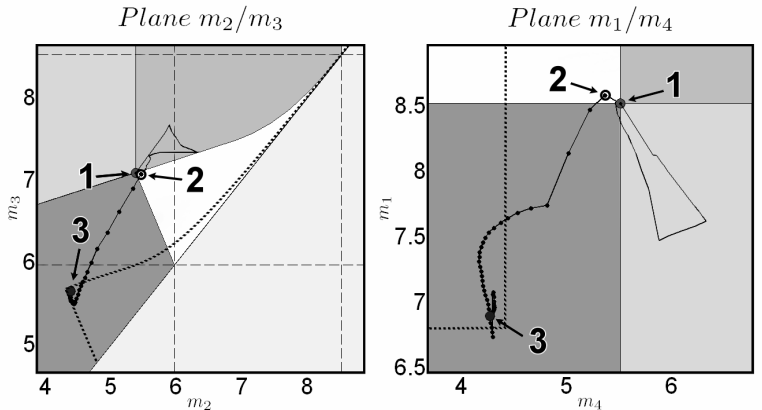

Fig. 11. Response of the system to a disturbance in Henry coefficients - cyclopentanone - cycloheptanone separation
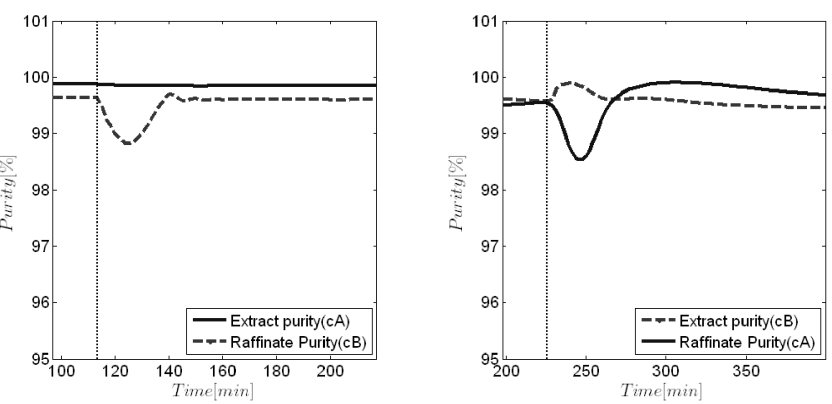

Fig. 12. Purities at the outlets before and after the disturbance (FOS - left; cyclopentanone - cycloheptanone - right)

The plant recovers from the perturbation in 15 cycles for the first separation and in 45 cycles for the second one.

In figure 12 a decrease less than $1.5 \%$ of the output purities can be observed. 


\section{CONCLUSIONS AND FUTURE WORK}

In this work, a simple adaptive controller is presented and extensively tested in simulation for two case studies including the separation of fructo - oligosaccharides (linear isotherms) and cyclopentanone - cycloheptanone (competitive Langmuir isotherms). The controller appears extremely robust and allows an optimization of the operating conditions even with very little knowledge on the adsorption characteristics. The price to pay is however the availability of sensors in each zone. Ongoing work is dedicated to the design and test of observers to allow a decrease in the number of sensors, and the experimental validation of the control strategy on a pilot plant (results will be reported at the time of conference).

\section{REFERENCES}

Engell, S. and Toumi, A. (2005). Optimisation and control of chromatography, Computers and Chemical Engineering 29(6), p. 1243-1252.

Erdem, G., Abel, S., Amanullah, M., Morari, M., Mazzotti, M. and Morbidelli, M. (2005). Automatic control of simulated moving beds - experimental verification, Adsorption 11(1), p. 573-577.

Fütterer, M. (2008). An Adaptive Control Concept for Simulated Moving Bed Plants in Case of Complete Separation, Chemical Engineering and Technology 31(10), p. 1438-1444.

Grosfils, V., Levrie, C., Kinnaert, M. and Wouwer, A.V. (2007). A systematic approach to SMB processes model identification from batch experiments, Chemical Engineering Science 62(15), pp. 3894-3908.

Grossmann, C., Erdem, G., Morari, M., Amanullah, M., Mazzotti, M. and Morbidelli, M. (2008). 'Cycle to cycle' Optimizing Control of Simulated Moving Beds, AIChE Journal 54(1), pp. 194-208.

Haag, J.,Wouwer, A.V., Lehoucq, S., Saucez, P. ( 2001). Modeling and simulation of a SMB chromatographic process designed for enantioseparation. Control Eng. Pract. 9 (8), p. 921-928.

Kawajiri, Y. and Biegler, L.T. (2006). A nonlinear programming superstructure for optimal dynamic operations of simulated moving processes, Industrial and Engineering Chemistry Research 45(25), p. 8503-8513.

Kawajiri, Y. and Biegler, L.T. (2006). Large scale nonlinear optimization for asymmetric operation and design of simulated moving beds, Journal of Chromatography A 1133, p. 226-240.

Kawajiri, Y. and Biegler, L.T. (2006). Optimization strategies for simulated moving bed and PowerFeed processes, AIChE Journal 52(4), p. 1343-1350.

Kawajiri, Y. and Biegler, L.T. (2008). Comparison of configurations of a four-column simulated moving bed process by multi-objective optimization, Adsorption Journal of the International Adsorption Society 14(2-3), p. 433-442.

Ludemann-Hombourger, O., Nicoud., R.M. and Bailly, M. (2000). The VariCol process: a new multicolumn continuous chromatographic process, Separation Science and Technology 35, p. 1829-1862.
Mazzotti, M., Storti, G. and Morbidelli, M. (1997). Optimal operation of simulated moving bed units for nonlinear chromatographic separations, Journal of Chromatography A 769(1), p. 3-24.

Nobre, C., Teixeira J.A. and Rodrigues L.R.(2012). Fructooligosaccharides purification from a fermentative broth using an activated charcoal column, New Biotechnology 29(3), pp. 395-401

Schiesser, W. E. (1996). Pde boundary conditions from minimum reduction of the pde. Applied Numerical Mathematics 20, p. 171-179.

Schramm, H., Grüner, S. and Kienle, A. (2003). Optimal operation of simulated moving bed chromatographic processes by means of simple feedback control, Journal of Chromatography A 1006(1-2), p. 3-13.

Schramm, H., Kaspereit, M., Kienle, A. and SeidelMorgenstern, A. (2003). Simulated moving bed process with cyclic modulation of the feed concentration, Journal of Chromatography A 1006, p. 77-86.

Song, I.H., Lee, S.B., Rhee, H.K. and Mazzotti, M. (2006). Optimization-based predictive control of a simulated moving bed process using an identified model, Chemical Engineering Science 61(18), p. 5179-6165.

Storti, G., Mazzotti, M., Morbidelli, M. and Carrà, S. (1993). Robust design of binary countercurrent adsorption separation processes, AIChE Journal 39(3), p. 471-492.

Tanimura, M., Tamura, M., Teshima, T., (1995). Japanese Patent 07-046097b.

Toumi, A., Engell, S., Ludemann-Hombourger, O., Nicoud, R.M. and Bailly, M. (2003). Optimization of simulated moving bed and VariCol processes, Journal of Chromatography A 1006, p. 15-31.

US Patent 2985 589. 1961.

Wouwer, A.V., Saucez, P. and Schiesser, W.E. (2004). Matlab - Based Method of Lines Toolbox, Available at www.matmol.org

Wouwer, A.V., Saucez, P. and Schiesser, W.E. (2004). Simulation of distributed parameter systems using a Matlab-based method of lines toolbox: Chemical engineering applications, Industrial and Engineering Chemistry Research 43(14), pp. 3469-3477.

Zhang, Z.Y., Mazzotti, M. and Morbidelli, M. (2003). Multiobjective optimization of simulated moving bed and VariCol processes using a genetic algorithm, Journal of Chromatography A 989(1), p. 95-108.

Zhang, Z.Y., Mazzotti, M. and Morbidelli, M. (2003). PowerFeed operation of simulated moving bed units: changing flow-rates during the switching interval, Journal of Chromatography A 1006(1-2), p. 87-99.

Zhang, Z.Y., Mazzotti, M. and Morbidelli, M. (2004). Continuous chromatographic processes with a small number of columns: comparison of simulated moving bed with VariCol, PowerFeed, and ModiCon, Korean Journal of Chemical Engineering 21(2), p. 454-464.

Zhang, Z.Y., Morbidelli, M. and Mazzotti, M. (2004). Experimental assessment of PowerFeed chromatography, AIChE Journal 50(3), p. 625-632. 